\title{
Performance of Indoor Residual Spray on entomological parameters of malaria transmission using pirimiphos-methyl in the context of pyrethroids resistance in Koulikoro region, Mali.
}

Moussa KEITA ( $D$ moussa@icermali.org )

CHERCHEUR

Nafomon SOGOBA

Malaria Research and Training Center

Boïssé Traoré

Malaria Research and Training Center

Fousseyni Kané

Malaria Research and Training Center

Boubacar Coulibaly

Malaria Research and Training Center

Sekou Fantamady Traoré

Malaria Research and Training Center

Seydou Doumbia

Malaria Research and Training Center

\section{Research}

Keywords: Mali, IRS, Long-lasting insecticidal nets, insecticide resistance, transmission

Posted Date: January 21st, 2020

DOI: https://doi.org/10.21203/rs.2.21391/v1

License: (a) (i) This work is licensed under a Creative Commons Attribution 4.0 International License.

Read Full License

Version of Record: A version of this preprint was published on April 1st, 2021. See the published version at https://doi.org/10.1016/j.actatropica.2020.105820. 


\section{Abstract}

Background: Following vectors resistance to both pyrethroid and carbamates, organophosphate (pirimiphos-methyl) was used in the Indoor Residual Spray campaigns of 2015 to 2016 in the district of Koulikoro. In this context, we assessed the effect of IRS on malaria transmission by comparing entomological indices in two localities: Koulikoro (LLINs+IRS), and Banamba (LLINs -only) districts.

Methods: The study was conducted in two villages of each of Koulikoro and Banamba in 2016. Pyrethroid spray catch and entry window trapping where used to collect mosquitoes monthly WHO bioassay test was used to assess mosquito's susceptibility to insecticides. Mosquitoes were identified into species by PCR and the infection rate using ELISA.

Results: An. coluzii was by far the most frequent species. Its density was rainfall dependent in no-IRS area, and almost independent in IRS area. The IR in the no-IRS area was $1.24 \%$, while in the IRS area, we could not detect infection. In the no-IRS area, the EIR was 0.21 infective bites /person month with the peak in September. High resistance to pyrethroids and carbamates and susceptibility to organophosphates was observed in all sites.

Conclusion: A big randomized study is needed to better estimate the added value of the IRS in an integrated malaria control strategy. Key words: Mali, IRS, Long-lasting insecticidal nets, insecticide resistance, transmission.

\section{Background.}

Long-lasting insecticidal nets (LLINs) and indoor residual spraying (IRS) are actively promoted as the main prevention strategy of malaria control and elimination plan [1]. The recent wide deployment of these two control tools, are considered to be responsible of the substantial reduction of the incidence and deaths related to the disease in Sub-sahara Africa $[2,3]$ Yet, the region continues to carry a disproportionately high share of the global burden of malaria deaths.

According to the 2015 Malaria Indicator Survey in Mali, the prevalence of malaria among children under five years of age was $36 \%$ at national level and $35 \%$ in the Koulikoro region[4]

In Mali, malaria still represents about $40 \%$ of the reasons for consultation [5]. The health services registered 2,369,245 cases of malaria including 686,017 severe cases. Children under 5 years are the most affected by the disease with $33.65 \%$ of cases, followed by pregnant women (4.95\%). The number of recorded deaths was 1978 , with a case fatality rate of $0.89 \%$ [6].

The national strategy for malaria control in Mali is based on four main interventions: i.) Early diagnosis and treatment by ACTs, ii.) Seasonal Malaria Chemoprevention (SMC) in children aged 3 to 59 months (SMC), iii) Preventive Intermittent Treatment (IPTp) with sulfadoxine-pyrimethamin (SP) in pregnant women and, iv) Vector control. Vector control relies heavily on LLINs at country level and IRS in only 
selected districts. With the emergence and recrudescence of the resistance of Anopheles gambiae sensu lato (An. gambiae s.l.) to insecticides the fragile progress made in malaria control can be compromised [7]. The wide and long term uses of pyrethroid insecticides in LLINs and in agriculture seem to be one of the main cause of increasing vector resistance to these products $[8,9]$.

As part of the strengthening of vector control strategies in the Koulikoro district, IRS was initiated by the National Malaria Control Program (NMCP) with the support of the US President's Malaria Initiative (PMI) since 2008. Pyrethroids (deltamethrin and lambda-cyhalothrin) were the first insecticides used in the IRS for the first 4 years [10] . Following resistance to these insecticides, they were replaced by carbamates (Bendiocarb) in 2011. Because of its short remanence, the carbamates (Bendiocarbe) were also replaced by the organophosphates (pirimiphos-methyl) in 2015. A mass distribution of LLINs was implemented in 2014 to achieve universal coverage of all populations at risk. Notwithstanding the deployment of all these strategies by the NMCP and its partners, no study was done so far to assess the effect of IRS campaigns on malaria transmission as measured by entomological inoculation rate in this context of vectors resistance to insecticides. In this study, we assessed the effect of IRS on malaria transmission by comparing entomological indices in two localities of the district of Koulikoro, where IRS was implemented for about 9 consecutive years, to similar two other villages of the its neighbored district of Banamba, where IRS was never be implemented.

\section{Materials And Methods.}

Study areas: This study was conducted in the villages of Karadie $(7.60 \mathrm{~W}, 13.24 \mathrm{~N})$ and Koula $(7.65 \mathrm{~W}$, $13.12 \mathrm{~N})$ in the district of Koulikoro and Kolondialan $(7.51 \mathrm{~W}, 13.49 \mathrm{~N})$ and N'Galamadibi $(7.48 \mathrm{~W}, 13.48 \mathrm{~N})$ in the district of Banamba (Figure 1). The climate in both districts is typical Sudano -sahelian savannah with two seasons: a long dry season from November to May and a wet season from june to october with a mean annual rainfall of $900-1200 \mathrm{~mm}$. The monthly mean temperature during the rainy season varies from 29 to $33^{\circ} \mathrm{C}$. An. gambiae s.l. is the predominant malaria vector (>98\%). Malaria transmission occurs mostly during the rainy season (June to October) with the mean monthly mosquito human biting rate reaching its peak in August and September.

LLINs, SMC for Children of 3 to 59 months old, and IPT with SP for pregnant women are control interventions implemented in both districts. Besides this, PMI has supported ten IRS campaigns in the districts of Koulikoro (2008 to 2016), while it has never been implemented in the district of Banamba. Agriculture, livestock and trade are the main economic activities.

Data collection: In each selected village, vector data were collected every month from June to November 2016 by Pyrethrum Spray Catch (PSC) and Entry Windows Traps (EWT).

Pyrethroid spray catches (PSC). In each study village, 30 sentinel houses were randomly selected from a list of total households. The sampling took into account the type of housing (e.g. thatch roof vs metal roof). Indoor-resting mosquitoes were collected by PSC which consisted of spraying pyrethrum in the 
selected houses between 8:00 to 11:00 in the morning. Two teams of 2 entomologists (a total of 4 ) were sampling 10-15 houses per day.

Entry window trapping (EWT). This method consisted of mounting on inside bedrooms windows the traps in order to trap the mosquitoes entering the rooms. Ten traps were mounted in ten randomly selected rooms. The catches took place during three successive nights per month. One of the local guides of the different villages was responsible for closing the traps very early in the morning (around 6:00 am) to prevent the trapped mosquitoes to come out with a small curtain sewn on the opening of the traps. The trapped mosquitoes were removed from the traps using a mouth aspirator, held in carton cups, and the number recorded on a data sheet.

Insecticide susceptibility bioassay (WHO, 2016). Anopheles gambiae larvae were collected in different types of breeding habitats in and around each site and reared to adults at the insectary ( $F_{0}$ generation). At the emergence, adult stages of 2-5 day-old young females were exposed to insecticides-impregnated papers (permethrin [0.75\%], deltamethrin [0.05\%] and pirimiphos-methyl [0.25\%]). Approximately 20-25 mosquitoes per tube with 2-6 replicates were exposed to diagnostic concentrations of insecticides on impregnated filter papers for 1 hour, and then transferred to a clean holding tube supplied with $10 \%$ sugar. Mortalities post exposures were determined after 24 hours according to who standard procedures[11]. All the tests were carried out at $27 \pm 1^{\circ} \mathrm{C}$ and a relative humidity of $70-80 \%$.

Samples processing. Collected mosquitoes were identified into species (An. gambiae s.s. An. coluzzii and An. arabiensis) by Polymerase Chain Reaction technique [12]. The sporozoïte infection rate [13] and human blood index (HBI) [14] were established using Enzyme Immuno-Sorbent Assay (ELISA) technique.

Statistical analysis. The data were entered in Excel and analyzed in SPSS version 22, STATA version 10, and GraphPad Prism 7. The following entomological parameters were calculated: vector density, man biting rate (MBR), sporozoite index (SI), entomological inoculation rate (EIR), human blood index $\mathrm{HBI}$ ) and parity rate (PR). The density of malaria vector was calculated as the average number of indoor resting mosquitoes per room; the MBR as the average number of mosquito bites (fed + half gravid over rooms' sleepers) per person per time unit; the SI corresponds to the percentage of anopheles of a given species carrying sporozoites; the $\mathrm{HBI}$ as the proportion of females mosquito having human blood in their guts; the PR was calculated as the proportion of parous females relative to the total number of mosquitoes dissected (Pare and nulliparous); and the EIR was calculated as the total number of infectious bites per human per time unit. The Pearson correlation test was used to determine the correlation between density and rainfall. The Chi-square test was used to compare the MBR, HBI, and PR.

Mosquito's mortality rates were calculated by dividing the number of survivor mosquitoes by the number exposed to the insecticide.

- Mortality in the range of $98-100 \%$ indicates susceptibility of the mosquitoes.

- A mortality of less than $98 \%$ is suggestive of the existence of resistance and further investigation is needed. 
- If mortality is less than $90 \%$, confirmation of the existence of resistant genes in the test population with additional bioassays is not necessary, provided that at least 100 mosquitoes of each species were tested.

\section{Results.}

A total of 2258 specimens were collected over the study period, 1988 by PSC and 270 mosquitoes by EWT. An. gambiae s.l. was the only malaria vector collected in both areas and consisted of three species: An. coluzzii, An arabiensis and An. gambiae s.s. An. coluzzii was by far the most frequent species in both IRS $(96 \%, n=97)$ and no-IRS $(95 \%, \mathrm{n}=430)$ areas.

An. gambiae s.I. density was increasing with rainfall in area with no-IRS, while it remained low regardless of the variations in rainfall in IRS area (Figure 3). The highest density was observed over July and August in the no-IRS area while in the IRS area, two small peaks were observed in July (just before the IRS implementation), and in September (two months after the IRS). There was a strong correlation between rainfall and An. gambiae s.I. density, with 1-month lag, and rainfall in both IRS $(R=0.888, P=0.018)$ and no-IRS $(R=0.806, P=0.053)$ areas.

The overall mean MMBRs of An. gambiae s.l. were higher in the no-IRS area (20.41 bites/person/month) than in the IRS area (3.03 bites/person/month). In the IRS area, the peak of MMB (7.47 bites/person/month) was observed in September (> 2 months after IRS) while in the no-IRS area the peak was observed in August (66 bites/persons/month). In both areas, the lowest biting rate was observed in June (Figure 4). The mean sporozoïte infection rate over the study period in the no-IRS area was $1.24 \%$ ( $n$ $=1670)$. In the IRS area, the number of An. gambiae s.I. collected and tested $(n=253)$ at the ELISA did not allow us to detect a sporozoïte infection. The average HBI over the study period was significantly lower $\left(X^{2}=19.84 ; P<0.001\right)$ in the IRS area $(74 \%, n=171)$ than in the no-IRS area $(86.9 \%, n=1199)$. In the no-IRS area, the overall mean EIR was 0.21 infective bites/person/month with its highest rate (0.75infective bites per person per month) being observed during September.

Results of WHO bioassay tests performed in each site with $\mathrm{F}_{0}$ progeny of adult female showed high resistance levels to pyrethroids (deltamethrin) followed by the carbamates (bendiocarb) in all study sites, and a fully susceptibility to organophosphates (pirimiphos-methyl) as shown in Figure 5.

\section{Discussion.}

In this study, we compared entomological indices of malaria transmission of the district of Koulikoro, where IRS was implemented for about 9 consecutive years, to its neighbored district of Banamba, where IRS was never been implemented. An. gambiae s.I. was the only malaria vector encountered in both study 
areas and consisted of An. coluzzii, An. arabiensis and An. gambiae (Figure 2). An. coluzzii, was the most prevailing species in both areas. Similarobservations have been made in several recent studies in Mali [15-17] and were explained by climatic conditions prevailing in the Sahel (temperature, humidity, etc.) which are favorable to the development of this species [18-20].

There was a synchronic variation in An. gambiae s.I. density and rainfall in the area of no-IRS, but not in the IRS area (Figure 3). Many studies have demonstrated the association between An. gambiae s.l. abundance and rainfall[21-23], especially in arid water-limited areas of Africa such as the Sahel, where the rain fed pools are heavily utilized by the dominant malaria vector species An. coluzzii [24]. Thus the absence of the synchronic variation in An. gambiae s.I. densities, MMBRs and rainfall in the IRS area is certainly due to the effect of the IRS. Indeed, mosquitoes were fully susceptible to the insecticide used in the IRS campaign of 2016, the pirimiphos-methyl, which also has an exito-repellent effect on mosquito preventing them to enter into sprayed houses.

In the IRS area, we could not detect any transmission as measured by EIR (Figure 4), while in the no-IRS area the transmission was typical seasonal with the peak observed at the end of the rainy season. The nonexistence of the seasonal peak in the IRS area can also be attributed to the effects of the insecticide used in the IRS. An. gambiae s.l. population was fully susceptible to the pirimiphos-methyl (Figure 5) which was used for the IRS campaign of 2016. This may has shortens the life expectancy of the mosquitoes to accomplish the complete parasite development in mosquito to ensure a transmission. It could also be that because of the exito-repellency effect of the pirimiphos-methyl and/or the collection method used (PSC and EWT).

\section{Conclusions.}

All entomological transmission parameters were lower in the IRS area compared to the no-IRS area and transmission was even undetectable in the IRS area. This observation shows the added values the IRS because mosquito population was resistant to pyrethroids which are used in LLINs (deltamethrin) but fully susceptible to organophosphates used in the 2016 IRS campaign (pirimiphos-methyl). A big randomized study is needed to better estimate the added value of the IRS in an integrated malaria control strategies and assess its cost-effectiveness.

\section{Abbreviations.}

Indoor residual spraying (IRS)

Long-lasting insecticidal nets (LLINs) 
Intermittent Preventive Treatment (IPT)

Seasonal Malaria Chemoprevention (SMC)

The Ministry of Health $(\mathrm{MOH})$

The National Malaria Control Program (NMCP)

Preventive Intermittent Therapy (IPT)

Case report forms (CRFs)

Rapid diagnostic test (RDT)

University of Sciences, Techniques and Technologies of Bamako (USTTB)

\section{Declarations}

\section{Ethical considerations}

The protocol of this project has been approved by the Ethics Committee of FMPOS/USTTB under the letter N02014/51/CE/FMPOS. The research activities related to this protocol were carried out in accordance with good clinical research practice in humans and good laboratory practice as set out in the international conventions (Helsinki Declaration; International Conference on the Harmonisation of Good Practice in Biomedical Research). All our researchers were trained in good clinical and laboratory practice during the research. In the field, the community (administrative, customary authorities) was informed of all aspects of the study.

Consent for publication: Not applicable

Availability of data and materials: The datasets generated during and/or analyzed during the current study are available from the corresponding author on reasonable request.

Competing interests: The authors declare that they have no competing interests

Funding: This work was funded by TDR/WHO under the grant B20388 with a support from the National Institutes of Allergy and Infectious Diseases (NIAID) through the West African International Center of Excellence for Malaria Research (ICEMR): NIAID U19 AI 089696 and U19 Al 129387. 
MK: has worked on the research hypothesis, data collection and analysis and write the manuscript.

NS: has worked on has worked on the research hypothesis and manuscript writing.

FK: has worked on data collection and sample processing.

BT: has worked on data collection and sample processing.

BC: has worked on data collection.

SFT: Correct and approved the latest version before submission.

NS: Correct and approved the latest version before submission.

SD: worked on the research hypothesis, correct and approved the latest version before submission

Acknowledgement. We thank the NMCP for their support and the population of the study sites for their collaboration. We also thank all MRTC staff who supported for the field work.

\section{References}

1. WHO: Global Malaria Programme \& World Health Organization. Malaria Unit. (2006). Indoor residual spraying : use of indoor residual spraying for scaling up global malaria control and elimination : WHO position statement. World Health Organization. https://apps.who.int/iris/handle/10665/69386 2006.

2. WHO: World Malaria Report 2016. Geneva: World Health Organization; 2016. Licence: CC BY-NC-SA 3.0 IGO. In.; 2016.

3. Bhatt S, Weiss DJ, Cameron E, Bisanzio D, Mappin B, Dalrymple U, Battle K, Moyes CL, Henry A, Eckhoff PA et al: The effect of malaria control on Plasmodium falciparum in Africa between $\mathbf{2 0 0 0}$ and 2015. Nature 2015, 526(7572):207-211.

4. PNLP: Programme National de Lutte contre le Paludisme (PNLP), Institut National de la Statistique (INSTAT), INFO-STAT, Institut National de la Recherche en Santé Publique (INRSP) et ICF International, 2016. Enquête sur les Indicateurs du Paludisme au Mali (EIPM) 2015. Rockville, Maryland, USA : INSTAT, INFO-STAT et ICF International. 2016.

5. SLIS: Evaluation du Système Local d'Information Sanitaire (SLIS) avec les Outils- PRISM. 20142014.

6. SLIS: Système Local d'Information Sanitaire (SLIS). Evaluation du Système local d'information Sanitaire SLIS 2015. 2015.

7. Keita M, Traore S, Sogoba N, Dicko AM, Coulibaly B, Sacko A, Doumbia S, Traore SF: [Susceptibility status of Anopheles gambiae sensu lato to insecticides commonly used for malaria control in Mali]. 
Bull Soc Pathol Exot 2016, 109(1):39-45.

8. Namountougou M, Simard F, Baldet T, Diabate A, Ouedraogo JB, Martin T, Dabire RK: Multiple insecticide resistance in Anopheles gambiae s.I. populations from Burkina Faso, West Africa. PLoS One 2012, 7(11):e48412.

9. Reid MC, McKenzie FE: The contribution of agricultural insecticide use to increasing insecticide resistance in African malaria vectors. Malar J 2016, 15:107.

10. Cisse MB, Keita C, Dicko A, Dengela D, Coleman J, Lucas B, Mihigo J, Sadou A, Belemvire A, George K et al: Characterizing the insecticide resistance of Anopheles gambiae in Mali. Malar J 2015, 14:327.

11. WHO: Test procedures for insecticide resistance monitoring in malaria vector mosquitoes (Second edition). 20 Avenue Appia, 1211 Geneva 27, Switzerland (tel.: +41 22 791 3264; fax: +41 22791 4857; email: bookorders@who.int). 2016.

12. Fanello C, Santolamazza F, della Torre A: Simultaneous identification of species and molecular forms of the Anopheles gambiae complex by PCR-RFLP. Med Vet Entomol 2002, 16(4):461-464.

13. Burkot TR, Zavala F, Gwadz RW, Collins FH, Nussenzweig RS, Roberts DR: Identification of malariainfected mosquitoes by a two-site enzyme-linked immunosorbent assay. Am J Trop Med Hyg 1984, 33(2):227-231.

14. Beier JC, Perkins PV, Wirtz RA, Koros J, Diggs D, Gargan TP, 2nd, Koech DK: Bloodmeal identification by direct enzyme-linked immunosorbent assay (ELISA), tested on Anopheles (Diptera: Culicidae) in Kenya. J Med Entomol 1988, 25(1):9-16.

15. Edillo FE, Toure YT, Lanzaro GC, Dolo G, Taylor CE: Spatial and habitat distribution of Anopheles gambiae and Anopheles arabiensis (Diptera: Culicidae) in Banambani village, Mali. J Med Entomol 2002, 39(1):70-77.

16. Edillo FE, Tripet F, Toure YT, Lanzaro GC, Dolo G, Taylor CE: Water quality and immatures of the $M$ and $\mathbf{S}$ forms of Anopheles gambiae s.s. and An. arabiensis in a Malian village. Malar J 2006, 5:35.

17. Sogoba N, Vounatsou P, Bagayoko MM, Doumbia S, Dolo G, Gosoniu L, Traore SF, Toure YT, Smith T: The spatial distribution of Anopheles gambiae sensu stricto and An. arabiensis (Diptera: Culicidae) in Mali. Geospat Health 2007, 1(2):213-222.

18. Toure YT, Petrarca V, Traore SF, Coulibaly A, Maiga HM, Sankare O, Sow M, Di Deco MA, Coluzzi M: The distribution and inversion polymorphism of chromosomally recognized taxa of the Anopheles gambiae complex in Mali, West Africa. Parassitologia 1998, 40(4):477-511.

19. Mbida Mbida A, Etang J, Akono Ntonga P, Eboumbou Moukoko C, Awono-Ambene P, Tagne D, Talipouo A, Ekoko W, Binyang J, Tchoffo R et al: [New insight into Anopheles coluzzii Coetzee \& Wilkerson, 2013 larval ecology in the Wouri estuary, Littoral-Cameroon]. Bull Soc Pathol Exot 2017, 110(2):92-101.

20. Keita M, Baber I, Sogoba N, Maiga HM, Diallo M, Doumbia S, Traore SF: [Vectorial transmission of malaria in a village along the Niger River and its fishing hamlet (Kenieroba and Fourda, Mali)]. Bull Soc Pathol Exot 2014, 107(5):356-368. 
21. Zhou G, Minakawa N, Githeko AK, Yan G: Association between climate variability and malaria epidemics in the East African highlands. Proc Natl Acad Sci U S A 2004, 101(8):2375-2380.

22. Koenraadt $C J$, Githeko AK, Takken W: The effects of rainfall and evapotranspiration on the temporal dynamics of Anopheles gambiae s.s. and Anopheles arabiensis in a Kenyan village. Acta Trop 2004, 90(2):141-153.

23. Kelly-Hope LA, Hemingway J, McKenzie FE: Environmental factors associated with the malaria vectors Anopheles gambiae and Anopheles funestus in Kenya. Malar J 2009, 8:268.

24. Bomblies A: Modeling the role of rainfall patterns in seasonal malaria transmission. Climatic Change 2012, 112(3-4):673-685.

\section{Figures}

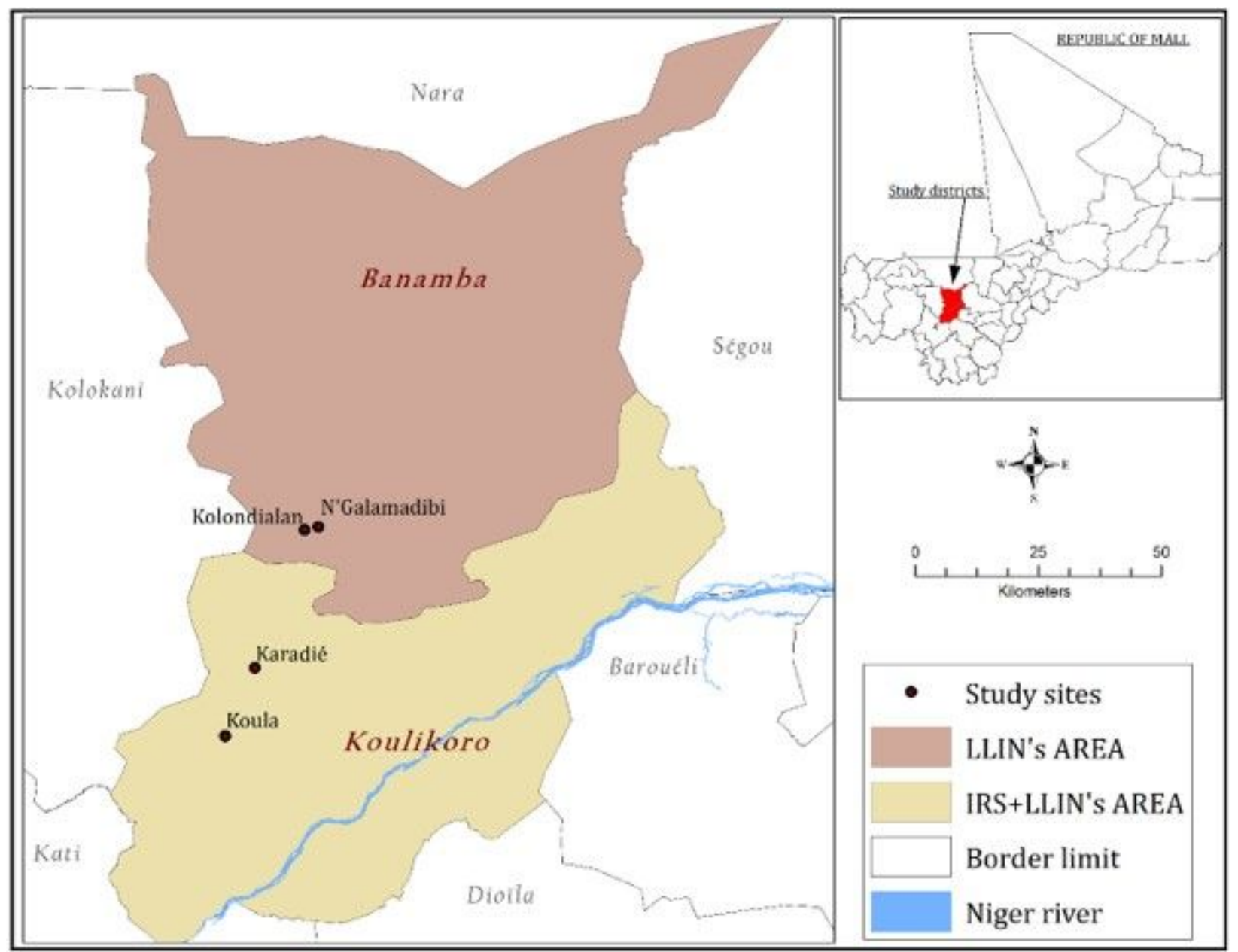

Figure 1

Map of Mali showing the different study sites 


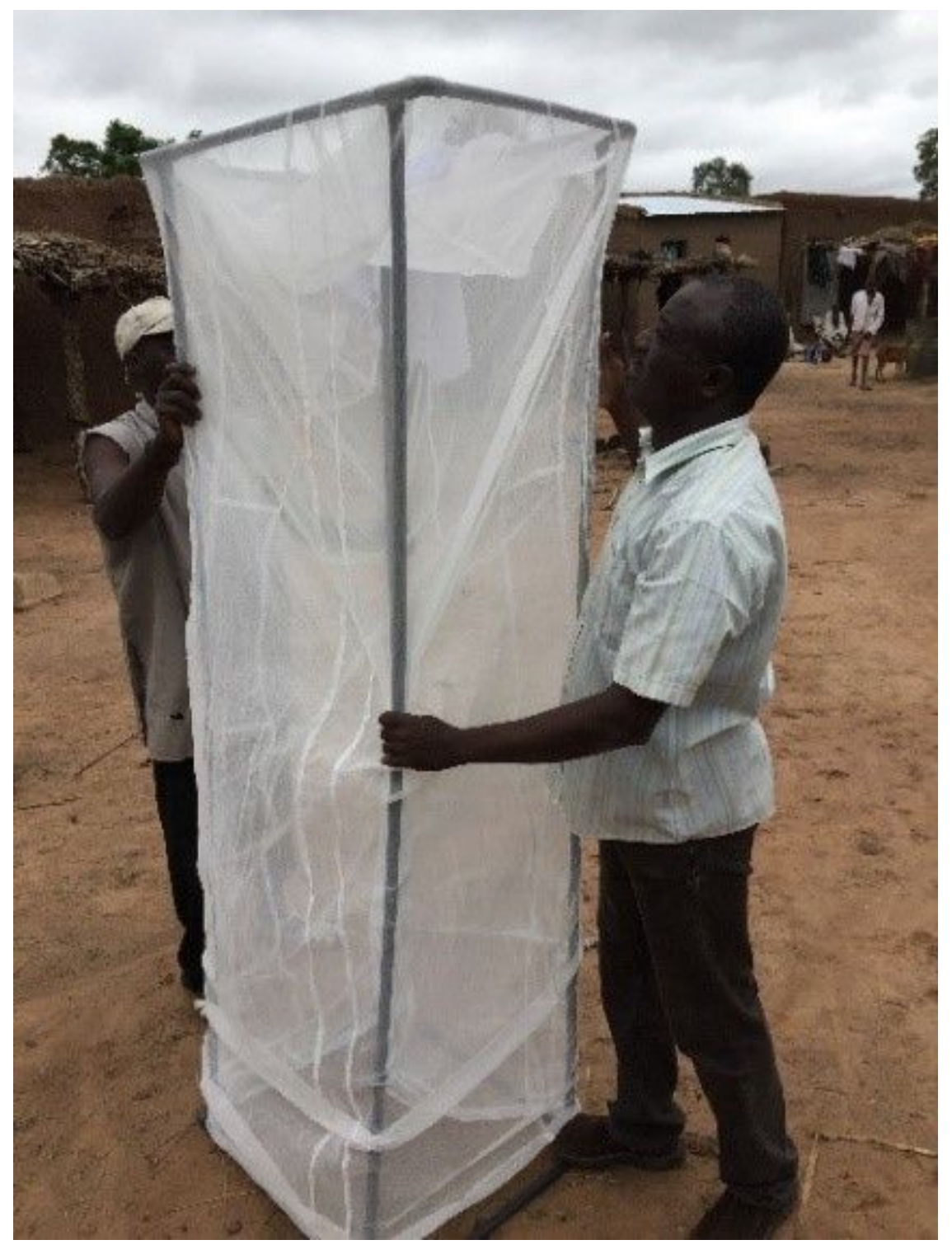

Figure 2

Field technician and local guide mounting a window trap 



$$
N=97
$$

$$
N=430
$$

\section{Figure 3}

An. gambiae s.I. species composition in the areas of IRS and n-IRS
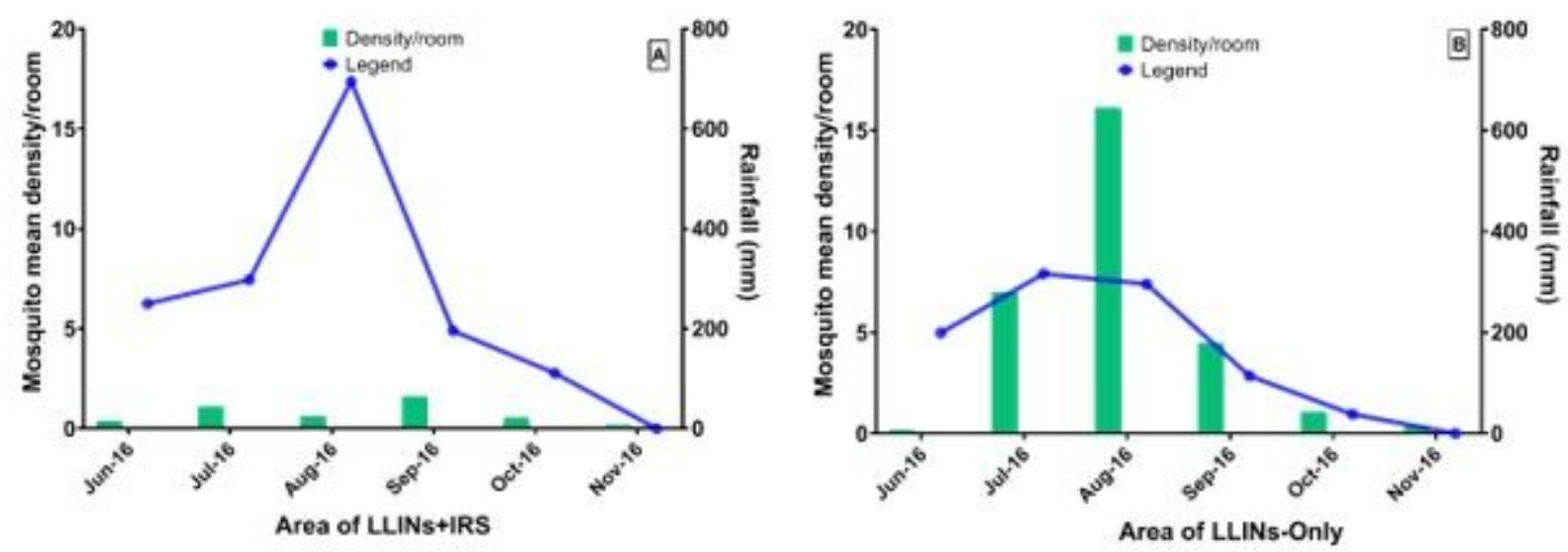

\section{Figure 4}

Variation in An. gambiae s.l. density (Red bars) and rainfall (blue line) in areas of IRS and no-IRS from June to November 2016. 



Figure 5: Monthly variation in An. gambiae s.l. man biting rates (green bars) and entomological inoculation rates (Red line) in areas of IIRS (A) and no-IRS (B) from June to November 2016.

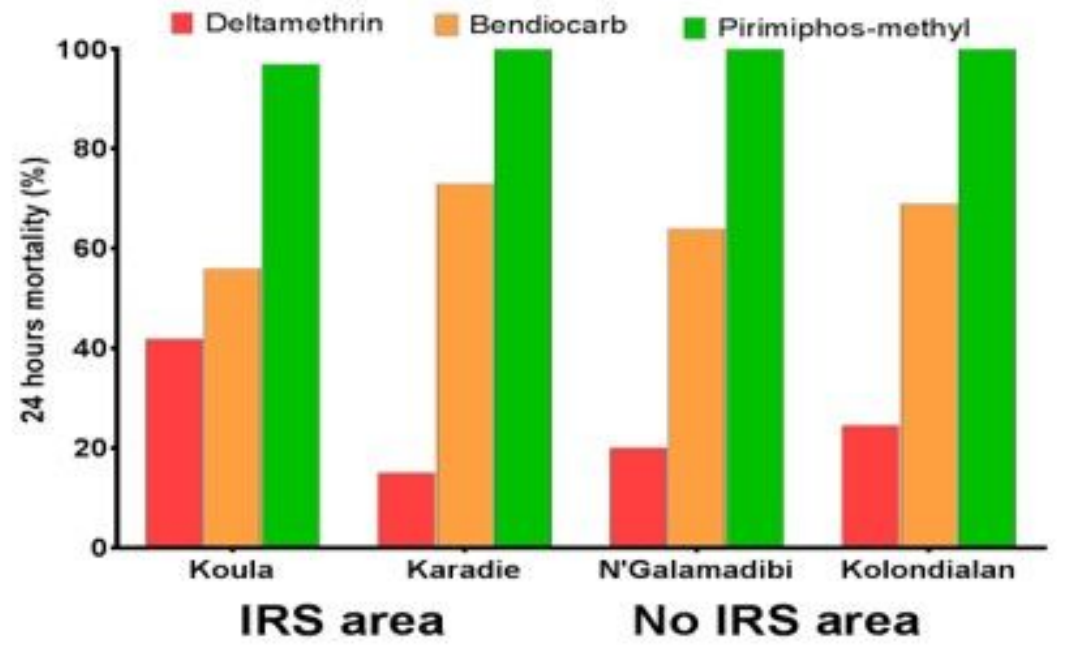

Figure 5

Monthly variation in An. gambiae s.l. man biting rates (green bars) and entomological inoculation rates (Red line) in areas of IIRS (A) and no-IRS (B) from June to November 2016. Observed 24 hours mortality (\%) of An. gambiae s.l. following $60 \mathrm{mn}$ exposition to Pyrethroids (Deltamethrin), Carbamates (Bendiocarb) and Organophosphates (Primiphos-methyl) in the selected study sites using WHO standard bioassay test. 